\title{
Cambios actitudinales y reducción del consumo de alcohol en adolescentes a partir de un programa de intervención psicosocial
}

\author{
Attitudinal changes and reduction of alcohol use in \\ adolescents following a psychosocial intervention program
}

María de la Villa Moral Jiménez ; Francisco Javier Rodríguez Díaz ${ }^{* *}$; Anastasio OveJero BernaL ${ }^{* \star}$; Carlos Sirvent RuIz ${ }^{\star \star \star \star}$.
* Área de Psicología Social.

** Área de Personalidad, Evaluación y Tratamiento Psicológico. Universidad de Oviedo.

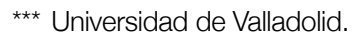

${ }^{\star \star \star \star}$ Fundación Instituto de Drogodependencias Spiral.

Enviar correspondencia a:

Francisco Javier Rodríguez Díaz. Facultad de Psicología.

Plaza de Feijóo, s/n - despacho 215. 33003 Oviedo (Asturias).

E-mail: gallego@uniovi.es

\section{RESUMEN}

La experimentación con alcohol representa una problemática que se va extendiendo entre el colectivo juvenil en los últimos años, produciéndose estabilizaciones en edades bajas de inicio al consumo. El objetivo es realizar un estudio exploratorio de las actitudes hacia el consumo de alcohol bajo una triple dimensionalidad (cognitiva, afectiva y comportamental) en una muestra de 755 alumnos de educación secundaria del Principado de Asturias (España). Posteriormente, de ellos se ha elegido una muestra de 141 adolescentes calificados de en riesgo, debido a sus actitudes de mayor permisividad en relación con su nivel de edad inferior (media=14.24 años) con objeto de aplicar un programa preventivo a nivel socioeducativo que fue evaluado con un intervalo temporal de dos, siete y doce meses.

Se ha comprobado la eficacia preventiva de la programación de actividades didácticas, un fortalecimiento de actitudes contrarias a la experimentación con alcohol y una mayor resistencia a la presión grupal, así como modificaciones en los patrones de ingesta y frecuencia de consumo. De los cuatro programas desarrollados (Intervención familiar, Experto, Información y Sensibilización básica) se comprueba la mayor eficacia preventiva de los programas competenciales y de intervención psicosocial en relación a los meramente informativos.

Palabras clave: Alcohol, adolescencia, actitudes hacia el consumo, prevención, intervención psicosocial.

\section{ABSTRACT}

Experimentation with alcohol constitutes a problem on the increase among young people in recent years, and mean age at first consumption has fallen. The goal of the present work was to carry out an exploratory study of attitudes toward alcohol use in terms of three dimensions (cognitive, affective and behavioural) in a sample of 755 secondary school pupils from the Principality of Asturias (Spain). Subsequently, we selected a sample of 141 adolescents described as at risk in view of their more permissive attitudes in relation to their lower age level (mean= 14.24 years), with the aim of applying a socio-educational preventive programme that included follow-up assessments at two, seven and twelve months.

The results showed the preventive efficacy of the didactic activities programmed, a strengthening of attitudes contrary to experimentation with alcohol, and greater resistance to peer pressure, as well as the modification of patterns and frequency of use. Of the four programs applied (Family Intervention, Expert, Information, and Basic Sensitization), we found greater preventive efficacy for the competence-enhancement and psychosocial intervention programs, compared to the merely informational type.

Key words: Alcohol, adolescence, attitudes toward consumption, prevention, psychosocial intervention. 


\section{INTRODUCCIÓN}

$\mathrm{E}$ consumo de alcohol sigue representando en la cultura mediterránea el uso de una droga asociada tradicionalmente a cultos báquicos, ritos grupales, sobreactivaciones lúdicas e imaginarios colectivos. En la literatura española especializada sobre el tema de los últimos años (Becoña y Míguez, 2006; Calafat, 2007; Calafat, Fernández, Juan y Becoña, 2007; Míguez y Becoña, 2008; Moral, 2007) se constata que este uso se hace extensivo a un amplio espectro del colectivo juvenil, vinculándolo a renovados modos de esparcimiento, como manifestaciones de una cultura recreativa nocturna de fin de semana, que se va imponiendo en espacios vivenciales compartidos con el grupo de iguales. Tales tendencias expansivas de la popularización del consumo de fin de semana responden a la modalidad calificada como "botellón" (Calafat, Juan, Becoña, Castillo, Fernández, Franco, Pereiro y Ros, 2004; Cortés, Espejo y Giménez, 2007, 2008; Franco, Juan, Pereriro, Calafat, Castillo y Becoña, 2005; Gómez-Fraguela, Fernández, Romero y Luengo, 2008) y a otras prácticas propias de la cultura recreativa juvenil (Bellis y Hughes, 2003; Moral y Ovejero, 2006; Sanders, 2006), entre otros indicadores básicos. Habitualmente tales prácticas van asociadas a consumos excesivos y concentrados de alcohol de acuerdo al patrón de consumo "binge-drinking" (atracón) (Anderson, 2007; Farke y Anderson, 2007) que se observan en toda Europa (Eurobarómetro, 2007).

En la experimentación juvenil con alcohol subyacen multitud de condicionantes de índole psicosocial, tales como deseos de integración grupal e intentos de evitación del rechazo, déficits de habilidades interpersonales ante presiones grupales, elicitaciones de liderazgo grupal, así como procesos de modelamiento simbólico (Moral, Rodríguez y Sirvent, 2005, 2006). Dada la vulnerabilidad metabólica, socioafectiva e identitaria de los adolescentes el tradicional calificativo de droga blanda aplicado a las experimentaciones con alcohol en absoluto responde a la realidad a consecuencia de los riesgos sobre la salud derivados de su ingesta abusiva, así como por constataciones relativas a una significativa reducción de las edades de inicio al tratamiento de la dependencia alcohólica (Arnau, 2001; Bach i Bach, 2000; Briñez-Horta, 2001; Casa, O'Ferrall y Vaca, 2001; Espada, Méndez e Hidalgo, 2000; Monrás, 2001). A ello se suman manifiestos cambios en los morfotipos de alcohólicos juveniles que incorporan nuevas modalidades de consumo y patrones de abuso, con sus propias patologias y problemas sobreañadidos, así como en las tipologías de jóvenes consumidores (compulsivo, toxicofílico, automedicativo, evasivo, desinhibitorio, socializante, sociopático, etc.) asociadas a politoxiconsumos varios (Espada, Méndez, Griffin y Botvin, 2003; Sirvent, Moral y Rodríguez, 2007). Asimismo, aunque en los últimos años ha descendido la proporción de consumidores de bebidas alcohólicas, ha aumentado la frecuencia de episodios de consumo intensivos (borracheras) y se ha estabilizado la edad de inicio al consumo de drogas institucionalizadas (alcohol y tabaco) entre los adolescentes españoles alrededor de los doce años y medio, de acuerdo con informes estatales (Delegación del Gobierno para el Plan Nacional
Sobre Drogas DGPNSD, 2003, 2004, 2005a, 2007). Según los datos del Informe sobre Alcohol de la DGPNSD (2007), en 2004 la edad media de inicio en el consumo de alcohol entre los estudiantes de 14 a 18 años fue de 13,7 años y de 15,1 años el inicio en el consumo semanal. En su conjunto, la tendencia global de consumo es indicativa de un estancamiento de la experimentación con drogas como tabaco y alcohol y el incremento de la experimentación con cannabis y cocaina, así como el ascenso de consumos de nuevas sustancias psicoactivas (éxtasis líquido, ketamina, enteógenos, etc.), tal como describe Becoña (2005).

Por lo que respecta al análisis de la esfera actitudinal, las actitudes hacia el consumo actúan como moduladoras de las experimentaciones, así como de los propios hábitos de ingesta. Suele hacerse mención al sistema de creencias y valores como marco de referencia común, de acuerdo a un sistema actitudinal integrado que determina la conducta, tal como fue conceptualizado hace décadas por Newcomb (1964). Aplicado a la experimentación con drogas, se emplea un enfoque multidimensional de las actitudes (Haddock y Zanna, 1999) basado en la naturaleza tridimensional de la actitud, según el cual tanto la información cognitiva como la afectiva son importantes en la predicción de las actitudes, de modo que afecto, cognición y comportamiento (sentir, saber y actuar) están conectados por una reacción valorativa de agrado/ desagrado. Aunque no se ha constatado una correspondencia biunívoca entre actitud y conducta, se confirma un estrecho vínculo entre las actitudes hacia las drogas y las conductas de consumo (Botvin y Botvin, 1992, 2000). De este modo, entre los jóvenes consumidores de alcohol suele constatarse una escasa conciencia del daño ocasionado, con mecanismos de distorsión en las percepciones de riesgo y actitudes de permisividad ante el consumo a nivel tanto cognitivo (creencias, expectativas, percepciones de riesgo, etc.), como afectivo (identificación con los usuarios, agrado o desagrado, etc.) y comportamental (disposición para el consumo e inclinación para la aceptación del hábito alcohólico) (Moral y Ovejero, 2003; Moral et al., 2006).

Asimismo, ha de incidirse a nivel teórico sobre las motivaciones que impulsan los consumos. Junto a la motivación hedónica, la presión normativa del grupo de iguales o la vinculada a la propia experimentación de sensaciones nuevas, el consumo de sustancias psicoactivas podría emplearse como vía de escape de la situación de moratoria psicosocial y cautividad en la adolescencia por la que atraviesan los jóvenes contemporáneos, evidenciada en diversos trabajos (CastiIlo, 1999; Moral y Ovejero, 1999, 2004; Ovejero, 2000). De acuerdo a una perspectiva psicosocial, se trataría de paliar sus crisis de identidad mediante la satisfacción plena del sentido otorgado al divertimento compartido, como tendencia de un renovado estilo de ocio juvenil. Para los jóvenes, la noche se convierte en el tiempo elegido, la calle en el espacio, el grupo de iguales en la compañía y lo lúdico en la principal motivación bajo manifestaciones de ritualizaciones grupales y fiestas compartidas (Delegación del Gobierno para el Plan Nacional sobre Drogas, 2006a, 2006b, 2006c). Evidentemente, semejantes prácticas en absoluto agotan otras 
muchas manifestaciones saludables de tipologías de jóvenes no identificados con las mismas y que desarrollan otro estilo de ocupación enriquecedora del tiempo libre de las diversas subculturas juveniles.

Ante la problemática descrita, ha de procederse a proponer líneas preventivas y de intervención. Se redunda en la necesidad de que desde las propuestas diseñadas al efecto se incida sobre un amplio espectro de agencias y poderes implicados, tales como la familia, el grupo de iguales, la escuela, los medios de comunicación, las instituciones y la comunidad, principalmente, cuestión ésta planteada en diversos programas de intervención psicosocial y sociocomunitaria (Legaza, 2002; Martín y Moncada, 2003; Moral y Ovejero, 2005a; Moradillo, 2003). Asimismo, en los últimos años, se han redoblado los esfuerzos institucionales en la planificación y ejecución de medidas preventivas y de intervención a través de la Estrategia Nacional sobre Drogas, vigente desde el año 2000 hasta el 2008, y con un Plan de Acción sobre Drogas (2005-2008) que comprende acciones centradas fundamentalmente en la prevención y la sensibilización social. Asimismo, el diseño e implementación de programas de educación preventiva incardinados dentro de estrategias de Educación para la Salud en las aulas con adolescentes escolarizados constituye una de las posibilidades de intervención ante esta problemática. Han de promoverse labores de integración de prevenciones específicas e inespecíficas, con diseño e implementación de programas de intervención psicosocial mediante los que se reestructuren las percepciones de riesgo asociadas a la experimentación, se doten de estrategias de afrontamiento frente a la presión grupal y se promuevan cambios actitudinales y de hábitos de consumo, que no se agoten exclusivamente en actuaciones meramente informativas, a lo cual han de sumarse actuaciones conjuntas de acción que potencien la corresponsabilización educativa, familiar y sociocomunitaria.

Descrito lo anterior, el propósito de la investigación es analizar los efectos preventivos sobre las actitudes y el consumo de alcohol de estrategias educativo-preventivas implementadas en diversos grupos experimentales. En concreto, en este estudio se persiguen una serie de objetivos básicos que se explicitan a continuación:

a) promover cambios en las actitudes hacia el consumo de alcohol en adolescentes, tanto iniciados al consumo como no experimentadores, en la dirección de un fortalecimiento de las actitudes contrarias al consumo o de una reducción de las disposiciones permisivas;

b) modificar la percepción de riesgo (creencias, expectativas, conocimientos, opiniones, etc.) sobre las consecuencias del consumo abusivo de alcohol;

c) incidir en la modificación de sus hábitos de consumo de alcohol; y, por último,

d) comprobar la eficacia preventiva de los programas de intervención psicosocial diseñados (Intervención Familiar, Grupo de Experto, Informativo y de Sensibilización básica), así como sus efectos diferenciales.

\section{MÉTODOS}

\section{Muestra}

Esta investigación ha sido diseñada como un proyecto con una orientación preventiva, de modo que con objeto de evaluar el fenómeno objeto de estudio y de priorizar las demandas se ha realizado un estudio descriptivo en el que se han seleccionado cuatro Centros de Enseñanza Secundaria del Principado de Asturias mediante un muestro intencional. En la primera encuestación participaron 755 alumnos del nivel de Secundaria (385 chicas, que representan el $51 \%$ de la muestra, y 370 chicos, $49 \%$ restante). Han sido elegidos mediante un muestro opinático, en función de la selección de varios grupos académicos por nivel escolar acorde con la disponibilidad de sus tutores para participar en la experiencia. Se ha seleccionado un estrato poblacional que abarca desde la primera adolescencia (12-13 años) y la adolescencia media (14-16 años) hasta edades más avanzadas (>16 años), siendo la edad de quince años la moda y la media se sitúa en los 14.69 años. La mayoría de los adolescentes provienen de un hogar nuclear, en el cual el nivel cultural paterno y materno es medio habiendo cursado estudios primarios completos un $61.2 \%$ y un $65.8 \%$, respectivamente; la principal cualificación profesional de los padres es obrero cualificado $(61.1 \%, n=347)$ y la principal labor profesional materna es la actividad sus labores $(76.0 \%, n=444)$, dedicándose el $6.8 \%$ $(n=40)$ a actividades comerciales y a otras tareas especializadas $(5.5 \%, n=32)$ y no especializadas $(5.8 \%, n=34)$ (véase Tabla 1).

Con posterioridad, se efectuó un muestreo de conglomerados con submuestreo (bietápico) con una selección de unidades elementales utilizando como conjuntos de referencia las clases seleccionadas en la primera etapa. Se ha empleado un diseño cuasiexperimental de diseño de grupo control no equivalente, en el que hay un grupo experimental (en esta oportunidad cuatro) y otro control, recibiendo pre-test y post-test. Esta submuestra de intervención calificada como de adolescentes en riesgo, fue seleccionada atendiendo a criterios básicos, tales como una tendencia actitudinal más permisiva en su conjunto que la del resto de participantes en la experiencia, disposiciones para el consumo y experimentaciones con alcohol y otras sustancias psicoactivas para unos niveles bajos de edad, de modo que se seleccionaron aquellos grupos académicos que cumplian estos criterios.

La muestra de intervención la componen 141 adolescentes seleccionados intencionalmente de la muestra total, de los cuales 76 son chicas (el $54.3 \%$ de la muestra) y 65 chicos (el $45.7 \%$ restante). Son alumnos adscritos a tercer curso de Educación Secundaria Obligatoria, curso con una significativa representación muestral en la muestra total seleccionada en primera instancia (alumnos de $3^{\circ}$ y $4^{\circ}$ de E.S.O., $n=242$ y $n=204$, respectivamente). La selección de estos grupos ha estado motivada por la presencia en ellos de adolescentes calificados como de riesgo por sus hábitos de consumo, ya instaurados en relación con su bajo nivel de edad. Esta muestra de intervención corresponde a la selección de cinco grupos académicos, cuatro de los cuales han participado como grupo experimental habiendo sido asignados alea- 
Tabla 1. Análisis descriptivo de la muestra total y de intervención.

\begin{tabular}{|c|c|c|c|c|c|c|c|c|c|c|c|c|}
\hline \multirow{3}{*}{ DISTRIBUCIÓN MUESTRAL } & \multicolumn{6}{|c|}{ Muestra Total $(\mathrm{N}=755)$} & \multicolumn{6}{|c|}{ Muestra de Intervención ( $\mathrm{N}=141)$} \\
\hline & \multicolumn{3}{|c|}{ VARÓN } & \multicolumn{3}{|c|}{ MUJER } & \multicolumn{3}{|c|}{ VARÓN } & \multicolumn{3}{|c|}{ MUJER } \\
\hline & \multicolumn{2}{|r|}{$\%$} & $\mathrm{~N}$ & $\%$ & $\mathrm{~N}$ & $\%$ & \multicolumn{2}{|r|}{$\%$} & $\mathrm{~N}$ & $\%$ & $\mathrm{~N}$ & $\%$ \\
\hline $\begin{array}{c}\text { Centro Experimental } 1 \\
\text { Grupo de Intervención familiar }\end{array}$ & 175 & 23,2 & 85 & 48,6 & 90 & 51,4 & 31 & 22,0 & 14 & 45,2 & 17 & 54,8 \\
\hline $\begin{array}{c}\text { Centro Experimental } 2 \\
\text { Grupo Informativo y Experto }\end{array}$ & 259 & 34,3 & 148 & 57,1 & 111 & 64,8 & 52 & 36,9 & 24 & 46,2 & 28 & 53,8 \\
\hline $\begin{array}{c}\text { Centro Experimental } 3 \\
\text { Grupo de Sensibilización básica }\end{array}$ & 165 & 21,9 & 79 & 50,6 & 77 & 49,4 & 30 & 21,3 & 15 & 51,9 & 13 & 48,1 \\
\hline Centro Control & 156 & 20,7 & 58 & 35,2 & 107 & 64,8 & 28 & 19,9 & 12 & 40,0 & 18 & 60,0 \\
\hline \multirow{3}{*}{ MUESTRA EDAD/SEXO } & \multicolumn{6}{|c|}{ Muestra Total } & \multicolumn{6}{|c|}{ Muestra de Intervención } \\
\hline & \multicolumn{3}{|c|}{ VARÓN } & \multicolumn{3}{|c|}{ MUJER } & \multicolumn{3}{|c|}{ VARÓN } & \multicolumn{3}{|c|}{ MUJER } \\
\hline & $\mathrm{N}$ & & $\%$ & $\mathrm{~N}$ & & $\%$ & $\mathrm{~N}$ & & $\%$ & $\mathrm{~N}$ & & $\%$ \\
\hline 12 años & 32 & & 8,7 & 22 & & 6,0 & 0 & & 0,0 & 0 & & 0,0 \\
\hline 13 & 48 & & 13,0 & 47 & & 12,8 & 3 & & 4,7 & 8 & & 10,8 \\
\hline 14 & 79 & & 21,4 & 96 & & 26,2 & 34 & & 51,6 & 49 & & 66,2 \\
\hline 15 & 109 & & 29,5 & 101 & & 27,6 & 23 & & 35,9 & 12 & & 16,2 \\
\hline 16 & 60 & & 16,3 & 65 & & 17,6 & 5 & & 7,8 & 5 & & 6,8 \\
\hline 17 & 30 & & 8,1 & 24 & & 6,6 & 0 & & 0,0 & 0 & & 0,0 \\
\hline 18 & 11 & & 3,0 & 11 & & 3,0 & 0 & & 0,0 & 0 & & 0,0 \\
\hline
\end{tabular}

toriamente a una de las cuatro modalidades de intervención explicitadas: Intervención Familiar ( $\mathrm{N}=31)$, Grupo de Experto $(\mathrm{N}=30)$, Informativo $(\mathrm{N}=22)$ y Grupo de Sensibilización básica $(\mathrm{N}=28)$ y uno como grupo control $(\mathrm{N}=30)$. De acuerdo con la distribución por niveles de edad se concreta que casi el sesenta por ciento $(58.7 \%, n=83)$ son adolescentes de catorce años, si bien el espectro es de los trece $(8.7 \%, n=12)$ a los dieciséis años $(7.2 \%, n=10)$. La elección de estos niveles de edad, ya justificada, es por tratarse de un periodo de edad en el que se suceden e interrelacionan cambios a múltiples niveles y se tiende a producir la iniciación al consumo.

\section{Variables Investigadas e Instrumentos de Evaluación}

De acuerdo a nuestro interés investigador se ha aplicado el Autoinforme de Consumo de Sustancias Psicoactivas (AICA) (Santacreu, Froján y Hernández, 1990, recogido por Santacreu y Froján, 1994) que consta de treinta items medidos en una escala Likert, con cinco niveles de respuesta (MA a MD). Analiza actitudes hacia el consumo de alcohol y otras drogas, de acuerdo a una triple dimensionalidad: cognitiva (creencias y expectativas), emocional (sentimientos y valoraciones) y comportamental (disposición para actuar), además de conocimientos sobre consumo de drogas, edad de inicio, historia de consumo, disponibilidad del producto en el entorno y modelos de consumidores (familia y grupo de iguales), del cual hemos seleccionado para los análisis aquellos items (AICA23-30) en los que se evalúan especificamente las variables relacionadas con el alcohol ya explicitadas. En la estructura factorial se ha obtenido el factor Actitud global hacia el consumo de alcohol ( $\Sigma$ AICA23-AICA30/8), que recoge las puntuaciones medias obtenidas en las ocho afirmaciones que exploran las actitudes de concienciación ante los efectos negativos del consumo de alcohol con afirmaciones en las que se analizan cuestiones relativas a la resistencia frente a la incitación al consumo, así como la escasa importancia otorgada a mostrar una conducta de abstinencia en un grupo de amigos consumidores (p.e. Aunque todos mis amigos beban yo no pienso hacerlo) y la ausencia de curiosidad para probar las drogas institucionalizadas (p.e. No tengo para probar el alcohol). Asimismo, se apela al daño social que ocasiona ( $E l$ alcohol es uno de los males de nuestra sociedad), manifestando un sentimiento contrario a su uso (Me disgusta que se consuma alcohol), valorando positivamente a quienes no son consumidores de alcohol (Admiro a quien no consume a/cohol) y desmitificando cuestiones asociadas a la creencia interesada en que su consumo en pequeñas cantidades no es perjudicial, entre otras. Se ha valorado el valor medio obtenido en la condición de Pre-Intervención y en las sucesivas evaluaciones de resultados. Por lo que respecta a las propiedades psicométricas de la escala en nuestra investigación se ha confirmado una adecuada validez interna y fiabilidad, hallándose puntuaciones elevadas (>.92) en el indicador de Cronbach (prueba de la buena consistencia interna), siendo el valor del coeficiente Alpha de .9314 para el total de los treinta items que componen el instrumento.

Los conocimientos que los adolescentes poseen sobre las consecuencias negativas derivadas de los abusos de la ingesta de alcohol, así como sus creencias y percepciones de riesgo han sido analizados empleando el Cuestionario de Información (Maciá y Méndez, 1988). Se ha empleado el factor Información sobre el consumo de alcohol ( INALCO1-INALC10/10) que exploran Creencias erróneas mitificadas y mentalidades del usuario, con distorsiones perceptivas de los riesgos y consecuencias derivados de la ingesta, creencias asociadas a la incitación al consumo y el primer contacto con las drogas y la evaluación de la mentalidad del usuario con ítems en los que se incide sobre mitos y falsas creencias muy extendidas popularmente, tales como las relativas a la determinación de lo social sobre la habituación posterior al alcohol (La mayor parte de los alcohólicos proceden de clases sociales bajas, INALC06), 
a la mentalidad del usuario (Que tanta gente consuma alcohol, demostraría que no es tan perjudicial como se dice, INALCO4) o, entre otras cuestiones, al carácter estimulante del alcohol ( $E$ I alcohol es un estimulante, no un depresor, INALC02), así como a los efectos dañinos a nivel físico y psicoemocional de la habituación al alcohol (El alcohol puede producir trastornos físicos y mentales, INALC01), entre otras cuestiones de interés. Se ha hallado una consistencia interna moderadamente baja (>.72) en relación con cada uno de los ítems (intensidad de la relación entre los elementos de la escala), en el caso particular del total se alcanzan valores cercanos a .75 .

La evaluación del componente comportamental de las actitudes se ha realizado mediante el Cuestionario de Actitudes hacia el alcohol, adaptado de Macià (1986) (Maciá y Méndez, 1988) e integrado por veinte ítems evaluados en formato Likert con cinco niveles de respuesta (MA a MD), mediante los que se evalúan las dimensiones valorativa (sentimientos asociados) y reactiva (inclinación para actuar) de las actitudes hacia el alcohol. A partir de la estructura factorial se ha obtenido el factor Disposición para el consumo de alcohol, mediante el que se analizan los valores medios hallados del análisis de los diez ítems en los que específicamente se evalúan las dimensiones actitudinales hacia el consumo de alcohol explicitadas con anterioridad ( $\Sigma$ ACT01-ACT10/10). Se evalúan las actitudes permisivas ante el consumo de alcohol y posible habituación alcohólica (Disposición para experimentar y habituarse al alcohol) con afirmaciones del tipo: Estaría dispuesto a ser consumidor habitual de alcohol (ACT02), Estaría dispuesto a comprar alcohol para mi uso (AСT03), Estaría dispuesto a tomar alcohol cuantas veces fuera necesario (ACT08) o Estaría dispuesto a convencer a mis amigos para que probasen el alcohol por primera vez (act09), entre otras, obviamente invirtiendo la tendencia de respuesta de aquellas planteadas en sentido contrario (p.e. Estaría dispuesto a mantener mi postura contraria en un grupo sobre el consumo de alcohol, ACT05; Estaría dispuesto a tratar de convencer a alguien sobre el riesgo de alcohol ACT07). Asimismo, la cantidad de alcohol ingerido y la frecuencia de consumo han sido medidas mediante el indicador HABICOL-92 (Beber01-18) (Pons y Buelga, 1995). El análisis de fiabilidad ofrece unos valores del coeficiente Alpha de Cronbach que oscilan entre .846 y .857 , correspondiendo un valor Alpha estandarizado moderadamente alto (.860).

\section{Procedimiento y Análisis de Datos}

Las fases de la investigación han sido: a) proceso de planificación en el ámbito comunitario y educativo; b) fase de sensibilización de distintos colectivos (educadores, padres, mediadores socioculturales, etc.); c) desarrollo propiamente de las labores de encuestación (recogida de información); d) fase de intervención psicosocial, con el desarrollo de un programa educativo-preventivo aplicado a cuatro grupos experimentales con las correspondientes modalidades que se explicitarán a continuación y, finalmente, e) evaluación de resultados a los dos, siete y doce meses de la aplicación del programa de prevención e intervención psicosocial.

La propuesta de intervención psicosocial con los cuatro grupos experimentales se pone en marcha a los tres meses de la primera encuestación, una vez analizados los datos aportados y seleccionada la correspondiente muestra de adolescentes en riesgo que integraron la muestra experimental descrita. Se emplearon ocho sesiones para el desarrollo del programa educativo-preventivo, con una periodicidad de una por semana, en el que se incluían actividades informativas varias sobre los mitos asociados al alcohol y la percepción de riesgo e ilusiones de invulnerabilidad de los usuarios, así como un entrenamiento en habilidades sociales y de resistencia a la incitación grupal al consumo y otras actividades sensibilizadoras, con discusiones grupales.

Los grupos experimentales fueron asignados aleatoriamente a una de las cuatro modalidades del programa de intervención desarrolladas por miembros del equipo investigador, en colaboración con los tutores de los alumnos:

a) en el Grupo de Intervención Familiar, junto a los contenidos comentados, se emplearon testimonios reales de personas alcohólicas y técnicas de discusión grupal (Philips 66) y se promovió una intervención paralela informativa con padres;

b) en el Grupo de Experto, en las sesiones impartidas por un especialista en drogodependencias, se potenció la labor informativa y sensibilizadora y actividades que redunden en la desmitificación de las creencias falsas sobre los usos/abusos de alcohol y sobre las percepciones de riesgo;

c) sesiones informativas impartidas por profesorado, entrenado al efecto, sin ninguna otra aportación fueron desarrolladas en el tercer Grupo Experimental (Grupo Informativo), con objeto de comprobar la prevista menor eficacia relativa de este tipo de programas genéricos con respecto a otros capacitadores; y, por último,

d) en el Grupo Experimental de Sensibilización básica se incluyeron sesiones de concienciación sobre los riesgos inherentes a los consumos con técnicas de reestructuración cognitiva y mediante el empleo de las técnicas de discusión grupal dirigidas por un profesional en drogodependencias, del equipo de investigación.

Finalmente, se ha realizado una evaluación de los efectos preventivos del programa de intervención psicosocial en distintos momentos temporales -a los dos meses de su implementación, a los siete y al año -, analizándose los cambios actitudinales y de hábitos de consumo en aquellos adolescentes con actitudes permisivas ante la experimentación y de fortalecimiento de la actitud de resistencia al consumo en otros. En este estudio se dará cuenta de los cambios hallados a nivel actitudinal, sobre modificación de las creencias y percepción de riesgo y en relación con la frecuencia de consumo de alcohol y la cantidad percibida de consumo semanal de alcohol, tras la propuesta de intervención. Los datos fueron recogidos de forma anónima, aunque con asignación de un código numérico (el número correspondiente a cada alumno en clase) y con el consentimiento informado de los participantes. El procesamiento y análisis estadístico de los datos (descriptivos, análisis factoriales y comparaciones de medias mediante la prueba $\mathrm{t}$ ) se ha llevado a cabo mediante el paquete estadístico S.P.S.S. versión 15.0. 


\section{RESULTADOS}

Se presentan los resultados obtenidos al comprobar la eficacia preventiva diferencial del programa educativo desarrollado con las peculiaridades especificadas para los cuatro grupos experimentales (Intervención Familiar, Grupo Experto, Grupo Informativo y Grupo de Sensibilización básica). Se han analizado los cambios favorables en las actitudes hacia el consumo de alcohol en la dirección de desarrollar y/o potenciar actitudes de resistencia ante la experimentación medida por el factor Actitud global hacia el consumo de alcohol contrastando el valor medio obtenido en la condición de PreIntervención con las sucesivas evaluaciones. De este modo, la Tabla 2 ofrece los resultados del análisis comparativo de las puntuaciones medias en los grupos experimentales y el grupo control en los periodos temporales en los que fue evaluada la investigación antes de la intervención y a los dos, siete y doce meses.
Se confirma la eficacia preventiva del programa de intervención psicosocial implementado en las cuatro modalidades, aunque conviene analizar a nivel didáctico las mejoras actitudinales por separado. En concreto, en la evaluación de los resultados a los dos meses de la intervención se ha confirmado la existencia de diferencias significativas en las actitudes globales hacia el alcohol en los cuatro grupos experimentales: Grupo de Intervención Familiar $(\mathrm{t}=4.022$, $\mathrm{p}<.0001)$, Experto $(\mathrm{t}=4.630, \mathrm{p}<.0001)$, Informativo $(\mathrm{t}=1.796$, $\mathrm{p}<.10)$ y de Sensibilización básica $(\mathrm{t}=1.470, \mathrm{p}<.05)$ en el sentido de un fortalecimiento de las actitudes contrarias al consumo tras la intervención. Esta tendencia se confirma por la obtención de puntuaciones medias más bajas asociadas a una tendencia actitudinal de mayor resistencia al consumo. Estas mejoras actitudinales, a su vez, también se confirman en la segunda evaluación de los resultados a los siete meses de la intervención (Post2) para el Grupo de Intervención Familiar $(\mathrm{t}=2.404, \mathrm{p}<.05)$, el Grupo Experto $(\mathrm{t}=1.611, \mathrm{p}<.05)$ y el de Sensibilización básica $(t=2.345, p<.05)$, si bien no alcanza

Tabla 2. Actitud global hacia el consumo de alcohol. Comparación de medias (prueba t para muestras relacionadas) entre los grupos experimentales y el control.

\begin{tabular}{|c|c|c|c|c|c|}
\hline ACTITUD CONSUMO & g.l. & Media & D.T. & $\mathrm{t}$ & p, 2 colas \\
\hline $\begin{array}{l}\text { Grupo Intervención Familiar } \\
\text { Actitud global alcohol Pre-Intervención } \\
\text { Post-Intervención POST1 (2 meses) }\end{array}$ & 27 & $\begin{array}{l}2,9598 \\
2,3259\end{array}$ & , 8865 & $4,022^{*}$ &, 000 \\
\hline $\begin{array}{l}\text { Actitud global alcohol Pre-Intervención } \\
\text { Post-Intervención POST2 (7 meses) }\end{array}$ & 27 & $\begin{array}{l}2,7366 \\
2,2188\end{array}$ & $\begin{array}{l}9553 \\
, 7141\end{array}$ & $2,404^{* * *}$ &, 023 \\
\hline $\begin{array}{l}\text { Actitud global alcohol Pre-Intervención } \\
\text { Post-Intervención POST3 (12 meses) }\end{array}$ & 27 & $\begin{array}{l}2,7143 \\
2,2500\end{array}$ & $\begin{array}{l}9 \\
9\end{array}$ & $2,003^{* * *}$ &, 055 \\
\hline $\begin{array}{l}\text { Grupo Experto } \\
\text { Actitud global alcohol Pre-Intervención } \\
\text { Post-Intervención POST1 ( } 2 \text { meses) }\end{array}$ & 26 & $\begin{array}{l}2,4722 \\
1,6343\end{array}$ & $\begin{array}{l}8301 \\
, 5370\end{array}$ & $4,630^{*}$ &, 000 \\
\hline $\begin{array}{l}\text { Actitud global alcohol Pre-Intervención } \\
\text { Post-Intervención POST2 (7 meses) }\end{array}$ & 25 & $\begin{array}{l}2,4087 \\
2,1020\end{array}$ & $\begin{array}{l}7629 \\
, 7616 \\
\end{array}$ & $1,611^{* * *}$ &, 012 \\
\hline $\begin{array}{l}\text { Actitud global alcohol Pre-Intervención } \\
\text { Post-Intervención POST3 (12 meses) }\end{array}$ & 25 & $\begin{array}{l}2,4095 \\
2,5000\end{array}$ & $\begin{array}{l}, 8470 \\
, 6606\end{array}$ &,- 543 &, 591 \\
\hline $\begin{array}{l}\text { Grupo Informativo } \\
\text { Actitud global alcohol Pre-Intervención } \\
\text { Post-Intervención POST1 (2 meses) }\end{array}$ & 16 & $\begin{array}{l}2,5147 \\
2,0662\end{array}$ & $\begin{array}{l}8557 \\
, 6805\end{array}$ & $1,796^{* * * *}$ &, 091 \\
\hline $\begin{array}{l}\text { Actitud global alcohol Pre-Intervención } \\
\text { Post-Intervención POST2 (7 meses) }\end{array}$ & 15 & $\begin{array}{l}2,6094 \\
2,4688\end{array}$ & $\begin{array}{l}, 7864 \\
, 5174\end{array}$ &, 565 &, 580 \\
\hline $\begin{array}{l}\text { Actitud global alcohol Pre-Intervención } \\
\text { Post-Intervención POST3 (12 meses) }\end{array}$ & 17 & $\begin{array}{l}2,5556 \\
2,5139\end{array}$ &, 8480 &, 172 &, 866 \\
\hline $\begin{array}{l}\text { Grupo Sensibilización básica } \\
\text { Actitud global alcohol Pre-Intervención } \\
\text { Post-Intervención POST1 ( } 2 \text { meses) }\end{array}$ & 25 & $\begin{array}{l}2,5288 \\
2,1477\end{array}$ & $\begin{array}{l}, 8271 \\
, 5947\end{array}$ & $1,470^{* * *}$ & .0154 \\
\hline $\begin{array}{l}\text { Actitud global alcohol Pre-Intervención } \\
\text { Post-Intervención POST2 (7 meses) }\end{array}$ & 21 & $\begin{array}{l}2,5852 \\
2,1477\end{array}$ & $\begin{array}{l}, 8508 \\
, 5947\end{array}$ & $2,345^{* * *}$ & 029 \\
\hline $\begin{array}{l}\text { Actitud global alcohol Pre-Intervención } \\
\text { Post-Intervención POST3 (12 meses) }\end{array}$ & 21 & $\begin{array}{l}2,5852 \\
2,0795\end{array}$ &, 8508 &, $872^{* *}$ & ,009 \\
\hline $\begin{array}{l}\text { Grupo CONTROL } \\
\text { Actitud global alcohol Pre-Intervención } \\
\text { Post-Intervención POST1 ( } 2 \text { meses) }\end{array}$ & 22 & $\begin{array}{l}2,5489 \\
3,3370\end{array}$ & $\begin{array}{l}\text {, } 1786 \\
, 7254\end{array}$ & $-4,362^{*}$ &, 000 \\
\hline $\begin{array}{l}\text { Actitud global alcohol Pre-Intervención } \\
\text { Post-Intervención POST2 (7 meses) }\end{array}$ & 19 & $\begin{array}{l}2,2375 \\
2,8625\end{array}$ & $\begin{array}{l}8875 \\
, 7790\end{array}$ & $-2,438^{* * *}$ &, 025 \\
\hline $\begin{array}{l}\text { Actitud global alcohol Pre-Intervención } \\
\text { Post-Intervención POST3 (12 meses) }\end{array}$ & 22 & $\begin{array}{l}2,3750 \\
2,7880\end{array}$ & $\begin{array}{l}8274 \\
, 7599\end{array}$ & $-2,074^{* * *}$ &, 049 \\
\hline
\end{tabular}

Actitud global hacia el consumo de alcohol $=[(\Sigma$ AICA23-AICA30) $/ 8]$

Actitud global alcohol Pre-Intervención = Actitud global hacia el consumo de alcohol antes del tratamiento experimental

${ }^{*} p<.001,{ }^{* *} p<.01,{ }^{* * *} p<.05$ 
significación estadística en el caso concreto del Grupo Informativo ( $\mathrm{t}=.565, \mathrm{p}=.580$ ). Por último, a raíz de la intervención se comprueba que el programa educativo-preventivo resulta eficaz para desarrollar y/o fortalecer tendencias actitudinales de resistencia al consumo, incluso después de un año de haberlo implementado (Post3), resultado confirmado para el Grupo de Intervención Familiar $(\mathrm{t}=2.003, \mathrm{p}<.05)$ y el Grupo de Sensibilización básica $(\mathrm{t}=2.872, \mathrm{p}<.01)$.

De la evaluación de la tendencia de cambio experimentada en la esfera actitudinal de los adolescentes que participaron como Grupo Control ha de apuntarse que se confirman cambios en el valor medio de las actitudes analizadas, en tanto que en la primera administración de las pruebas se obtuvo una puntuación media de $2.5489(\mathrm{~N}=22)$, en comparación con el resultado obtenido algunos meses después, destacando su incremento significativo hacia una mayor permisividad ante el consumo (Media $=3.3370, t=-4.362$, $\mathrm{p}<.0001)$. Esta tendencia se mantiene a lo largo de las sucesivas evaluaciones, tanto a los siete meses $(t=-2.438, p<.05)$ como al año $(t=-2.074, p<.05)$. Ello representa un incremento de las actitudes favorables hacia la experimentación con alcohol, lo cual es indicativo de la tendencia natural entre los adolescentes a mostrar progresivamente actitudes (creencias, percepción valorativa e inclinación para el consumo) más permisivas ante el consumo, así como una mayor valora- ción de la experimentación en sí y una disposición actitudinal asociada a tales creencias, en virtud de la que se predispone al consumo de un modo preferencial.

Tomando el factor Disposición global hacia el consumo de alcohol como referencia se ha efectuado una Comparación de medias (prueba t para muestras relacionadas) entre los grupos experimentales y el control, con objeto de verificar la existencia de diferencias significativas en las actitudes de los adolescentes entre la primera evaluación pre-intervención y las sucesivas para determinar en cuáles de los grupos de intervención y en qué intervalos temporales se manifiestan los efectos del programa educativo-preventivo (véase Tabla 3). En este sentido, han alcanzado significación las puntuaciones medias del Grupo de Sensibilización básica medidas a los dos meses de finalizar el programa de intervención $(\mathrm{t}=3.097, \mathrm{p}<.01)$ y el Grupo de Intervención familiar en la segunda evaluación de los resultados $(t=1.454, p<.05)$, en la dirección de indicar un fortalecimiento de la tendencia de resistencia frente a la experimentación con alcohol. En el resto de condiciones experimentales e intervalos temporales las diferencias halladas no han tenido significación estadistica. En esta ocasión, el análisis se ha centrado en el componente comportamental de las actitudes hacia el consumo de alcohol que se comprueba que es menos modificable que las creencias o las percepciones valorativas, ya analizadas.

Tabla 3. Disposición global ante la experimentación con alcohol. Comparación de medias (prueba t para muestras relacionadas) entre los grupos experimentales y el control

\begin{tabular}{|c|c|c|c|c|c|}
\hline ACTITUD CONSUMO & g.l. & Media & D.T. & $\mathrm{t}$ & p, 2 colas \\
\hline $\begin{array}{l}\text { Grupo Intervención Familiar } \\
\text { Disposición consumo de alcohol Pre-Intervención } \\
\text { Post-Intervención POST1 ( } 2 \text { meses) }\end{array}$ & 27 & $\begin{array}{l}3,3036,4 \\
2,2429,5\end{array}$ &, 4803 &, 500 &, 621 \\
\hline $\begin{array}{l}\text { Disposición consumo de alcohol Pre-Intervención } \\
\text { Post-Intervención POST2 (7 meses) }\end{array}$ & 27 & $\begin{array}{l}3,2286,5 \\
3,0750,3\end{array}$ & ,5054 & $1,954^{* * *}$ &, 0157 \\
\hline $\begin{array}{l}\text { Disposición consumo de alcohol Pre-Intervención } \\
\text { Post-Intervención POST3 ( } 12 \text { meses) }\end{array}$ & 27 & $\begin{array}{l}3,2000,4 \\
3,1929,3\end{array}$ & $\begin{array}{l}4 \\
, 4439 \\
, 3731\end{array}$ &, 065 & 949 \\
\hline $\begin{array}{l}\text { Grupo Experto } \\
\text { Disposición consumo de alcohol Pre-Intervención } \\
\text { Post-Intervención POST1 ( } 2 \text { meses) }\end{array}$ & 25 & $\begin{array}{l}3,1308,3 \\
3,0885,5\end{array}$ &, 3937 &, 345 & ,733 \\
\hline $\begin{array}{l}\text { Disposición consumo de alcohol Pre-Intervención } \\
\text { Post-Intervención POST2 (7 meses) }\end{array}$ & 25 & $\begin{array}{l}3,1192,3 \\
3,2885,6\end{array}$ & , 3940 & $-1,057$ &, 301 \\
\hline $\begin{array}{l}\text { Disposición consumo de alcohol Pre-Intervención } \\
\text { Post-Intervención POST3 ( } 12 \text { meses) }\end{array}$ & 28 & $\begin{array}{l}3,1310,3 \\
3,0690,4\end{array}$ & , 3761 &, 601 &, 552 \\
\hline $\begin{array}{l}\text { Grupo Informativo } \\
\text { Disposición consumo de alcohol Pre-Intervención } \\
\text { Post-Intervención POST1 ( } 2 \text { meses) }\end{array}$ & 16 & $\begin{array}{l}3,0000,3 \\
2,8765,2\end{array}$ & ,3260 & 1,692 &, 110 \\
\hline $\begin{array}{l}\text { Disposición consumo de alcohol Pre-Intervención } \\
\text { Post-Intervención POST2 (7 meses) }\end{array}$ & 15 & $\begin{array}{l}3,0250,3 \\
3,1688 \\
\end{array}$ & $\begin{array}{l}3194 \\
, 2983 \\
\end{array}$ &,- 1237 &, 235 \\
\hline $\begin{array}{l}\text { Disposición consumo de alcohol Pre-Intervención } \\
\text { Post-Intervención POST3 (12 meses) }\end{array}$ & 17 & $\begin{array}{l}2,9556,3 \\
3,0667,1\end{array}$ & $\begin{array}{l}3681 \\
, 1970\end{array}$ & $-1,411$ & ,176 \\
\hline $\begin{array}{l}\text { Grupo Sensibilización básica } \\
\text { Disposición consumo de alcohol Pre-Intervención } \\
\text { Post-Intervención POST1 ( } 2 \text { meses) }\end{array}$ & 25 & $\begin{array}{l}3,1174,6 \\
2,7000\end{array}$ & $\begin{array}{l}6148 \\
, 4710\end{array}$ & $3,097^{* *}$ &, 005 \\
\hline $\begin{array}{l}\text { Disposición consumo de alcohol Pre-Intervención } \\
\text { Post-Intervención POST2 ( } 7 \text { meses) }\end{array}$ & 21 & $\begin{array}{l}3,0136, \\
3,0045,5\end{array}$ & $\begin{array}{l}4 \\
, 4179 \\
, 5744\end{array}$ &, 057 &, 955 \\
\hline $\begin{array}{l}\text { Disposición consumo de alcohol Pre-Intervención } \\
\text { Post-Intervención POST3 (12 meses) }\end{array}$ & 21 & $\begin{array}{l}3,0136 \\
3,0227\end{array}$ & $\begin{array}{l}4179 \\
, 3294\end{array}$ &,- 081 &, 936 \\
\hline $\begin{array}{l}\text { Grupo CONTROL } \\
\text { Disposición consumo de alcohol Pre-Intervención } \\
\text { Post-Intervención POST1 ( } 2 \text { meses) }\end{array}$ & 22 & $\begin{array}{l}2,9769,4 \\
3,1231,5\end{array}$ & $\begin{array}{l}.4990 \\
, 5094\end{array}$ &,- 899 &, 377 \\
\hline $\begin{array}{l}\text { Disposición consumo de alcohol Pre-Intervención } \\
\text { Post-Intervención POST2 (7 meses) }\end{array}$ & 19 & $\begin{array}{l}3,2650,7 \\
3,0800,3\end{array}$ & , 7471 & 1.082 & ,293 \\
\hline $\begin{array}{l}\text { Disposición consumo de alcohol Pre-Intervención } \\
\text { Post-Intervención POST3 (12 meses) }\end{array}$ & 19 & $\begin{array}{l}3,1130,6 \\
2,9870,4\end{array}$ & $\begin{array}{l}6580 \\
6864\end{array}$ & ,740 & ,467 \\
\hline
\end{tabular}

Disposición para el consumo de alcohol= $[(\Sigma$ ACT01-ACT10)/10]

${ }^{*} p<.001,{ }^{* *} p<.01,{ }^{* * *} p<.05$ 
Tabla 4. Creencias e Información global sobre el alcohol y sus efectos. Comparación de medias entre los grupos experimentales y el control (prueba t para muestras relacionadas).

\begin{tabular}{|c|c|c|c|c|c|}
\hline ACTITUD CONSUMO & g.l. & Media & D.T. & $\mathrm{t}$ & p. 2 colas \\
\hline $\begin{array}{l}\text { Grupo Intervención Familiar } \\
\text { Información global sobre alcohol Pre-Intervención } \\
\text { Post-Intervención POST1 (2 meses) }\end{array}$ & 27 & $\begin{array}{l}1,7857 \\
1,5321\end{array}$ & $\begin{array}{l}.4325 \\
.2830\end{array}$ & $1,955^{* *}$ & ,045 \\
\hline $\begin{array}{l}\text { Información global sobre alcohol Pre-Intervención } \\
\text { Post-Intervención POST2 (7 meses) }\end{array}$ & 27 & $\begin{array}{l}1,7964 \\
1,7643\end{array}$ & $\begin{array}{l}.4316 \\
, 2482\end{array}$ & 368 &, 716 \\
\hline $\begin{array}{l}\text { Información global sobre alcohol Pre-Intervención } \\
\text { Post-Intervención POST3 (12 meses) }\end{array}$ & 27 & $\begin{array}{l}1,8179 \\
1,5926\end{array}$ & $\begin{array}{l}.4154 \\
.2900\end{array}$ & $1,892^{* * *}$ &, 069 \\
\hline $\begin{array}{l}\text { Grupo Experto } \\
\text { Información global sobre alcohol Pre-Intervención } \\
\text { Post-Intervención POST1 (2 meses) }\end{array}$ & 25 & $\begin{array}{l}1,7222 \\
1,5926\end{array}$ & $\begin{array}{l}2900 \\
, 3075\end{array}$ & 1,592 & 733 \\
\hline $\begin{array}{l}\text { Información global sobre alcohol Pre-Intervención } \\
\text { Post-Intervención POST2 (7 meses) }\end{array}$ & 25 & $\begin{array}{l}1,7346 \\
1,6269\end{array}$ & $\begin{array}{l}, 2741 \\
, 2324\end{array}$ & 1,535 & 301 \\
\hline $\begin{array}{l}\text { Información global sobre alcohol Pre-Intervención } \\
\text { Post-Intervención POST3 (12 meses) }\end{array}$ & 28 & $\begin{array}{l}1,7241 \\
1,6034\end{array}$ & $\begin{array}{l}, 2824 \\
, 3510\end{array}$ & 1,638 &, 552 \\
\hline $\begin{array}{l}\text { Grupo Informativo } \\
\text { Información global sobre alcohol Pre-Intervención } \\
\text { Post-Intervención POST1 (2 meses) }\end{array}$ & 16 & $\begin{array}{l}1,6412 \\
1,6765\end{array}$ & $\begin{array}{l}, 3318 \\
, 2513\end{array}$ &,- 367 & ,719 \\
\hline $\begin{array}{l}\text { Información global sobre alcohol Pre-Intervención } \\
\text { POST2 (7 meses) }\end{array}$ & 15 & $\begin{array}{l}1,6500 \\
1,6563\end{array}$ & $\begin{array}{l}.3406 \\
.4082\end{array}$ &,- 057 &, 955 \\
\hline $\begin{array}{l}\text { Información global sobre alcohol Pre-Intervención } \\
\text { Post-Intervención POST3 (12 meses) }\end{array}$ & 17 & $\begin{array}{l}1,6500 \\
1,7000\end{array}$ & $\begin{array}{l}, 3240 \\
, 3378\end{array}$ &,- 651 & ,524 \\
\hline $\begin{array}{l}\text { Grupo Sensibilización básica } \\
\text { Información global sobre alcohol Pre-Intervención } \\
\text { Post-Intervención POST1 (2 meses) }\end{array}$ & 25 & $\begin{array}{l}1,7731 \\
1,6385 \\
\end{array}$ & $\begin{array}{l}, 3131 \\
, 3600\end{array}$ & $1,573^{* *}$ &, 012 \\
\hline $\begin{array}{l}\text { Información global sobre alcohol Pre-Intervención } \\
\text { Post-Intervención POST2 (7 meses) }\end{array}$ & 21 & $\begin{array}{l}1,7818 \\
1,7273\end{array}$ & $\begin{array}{l}, 3361 \\
, 2711\end{array}$ & ,552 & ,587 \\
\hline $\begin{array}{l}\text { Información global sobre alcohol Pre-Intervención } \\
\text { Post-Intervención POST3 (12 meses) }\end{array}$ & 21 & $\begin{array}{l}1,7818 \\
1,8136\end{array}$ & $\begin{array}{l}, 3361 \\
, 2455\end{array}$ &,- 425 & ,675 \\
\hline $\begin{array}{l}\text { Grupo CONTROL } \\
\text { Información global sobre alcohol Pre-Intervención } \\
\text { Post-Intervención POST1 (2 meses) }\end{array}$ & 22 & $\begin{array}{l}1,7826 \\
1,7957\end{array}$ & $\begin{array}{l}, 3761 \\
, 3364\end{array}$ &,- 138 & ,892 \\
\hline $\begin{array}{l}\text { Información global sobre alcohol Pre-Intervención } \\
\text { Post-Intervención POST2 (7 meses) }\end{array}$ & 19 & $\begin{array}{l}1,7700 \\
1,7450\end{array}$ & $\begin{array}{l}, 2754 \\
, 2928\end{array}$ & ,341 & 737 \\
\hline $\begin{array}{l}\text { Información global sobre alcohol Pre-Intervención } \\
\text { Post-Intervención POST3 (12 meses) }\end{array}$ & 22 & $\begin{array}{l}1,7696 \\
1,7043\end{array}$ & $\begin{array}{l}2670 \\
, 2977\end{array}$ & 1,084 & .290 \\
\hline
\end{tabular}

Información global sobre alcohol=[( INALCO1-INALC10)/10]

${ }^{*} p<.01,{ }^{* *} p<.05{ }^{* * *} p<.10$

Como en los casos anteriores descritos, se ha realizado un análisis de comparación de medias en los valores hallados en los grupos experimentales y control en la primera encuestación, y en las sucesivas evaluaciones de los resultados, una vez finalizada la programación de actividades didácticas de carácter preventivo en el factor Creencias e Información global sobre el alcohol y sus efectos (véase Tabla 4). Las diferencias se concentran en el Grupo de Intervención familiar en la evaluación efectuada a los dos meses $(t=1.755, p<.05)$ y se mantienen al ser evaluadas al año de la intervención en el sentido predicho de fortalecimiento del componente cognitivo de la actitud, manifestando los adolescentes unas creencias menos mitificadas y mayores percepciones de riesgo.

Finalmente, se ofrecen los resultados obtenidos al medir la frecuencia de alcohol ingerido reportada por los adolescentes integrantes de la muestra total en la primera encuestación y en las muestras de intervención en las sucesivas evaluaciones de los resultados. Se reportan consumos referentes a la cantidad de alcohol consumida regularmente cada semana, que se adopta como referencia comparativa de aquellos usos que reconocen los adolescentes de los cuatro grupos de intervención (cuya edad media recordemos que es significativamente inferior a la de la muestra total) y cuyos hábitos de consumo se evalúan mediante el análisis de la cantidad de alcohol consumida de manera habitual (véase Tabla 5). Se comprueba que aún cuando la mayoría de los adolescentes no consumen regularmente alcohol, sin embargo los consumos efectuados se concentran en una periodicidad de uno a tres días por semana para la mayor parte de las sustancias con graduación alcohólica incluidas en el análisis. A su vez, resulta preocupante que un 15.8\% $(\mathrm{N}=93)$ de los adolescentes confiesen que consumen combinados a diario, así como que casi un veinte por ciento $(19.2 \%, \mathrm{~N}=113)$ compartan litronas también a diario. 
Tabla 5. Hábito de consumo semanal de Alcohol en la muestra total en la primera recogida de datos $(\mathrm{N}=755)$.

\begin{tabular}{|c|c|c|c|c|c|c|c|c|}
\hline \multirow{2}{*}{ CONSUMO DE ALCOHOL } & \multicolumn{2}{|c|}{ NADA } & \multicolumn{2}{|c|}{ 1-3 días/semana } & \multicolumn{2}{|c|}{ 4-6 días/semana } & \multicolumn{2}{|c|}{$>6$ días/semana } \\
\hline & $\%$ & $\mathrm{~N}$ & $\%$ & $\mathrm{~N}$ & $\%$ & $\mathrm{~N}$ & $\%$ & $\mathrm{~N}$ \\
\hline Vino (vasos) & 89,1 & 525 & 8,0 & 47 & 1,2 & 7 & 1,7 & 10 \\
\hline Cañas & 63,3 & 373 & 22,9 & 135 & 8,0 & 47 & 5,8 & 34 \\
\hline Botellin & 80,6 & 475 & 11,4 & 67 & 4,8 & 28 & 3,2 & 19 \\
\hline Jarras Cerveza & 89,8 & 529 & 3,4 & 20 & 3,9 & 23 & 2,9 & 17 \\
\hline Carajillos & 90,7 & 534 & 4,2 & 25 & 1,9 & 11 & 3,2 & 19 \\
\hline Combinados & 48,7 & 287 & 18,0 & 106 & 17,5 & 103 & 15,8 & 93 \\
\hline Licores & 72,0 & 424 & 13,1 & 77 & 7,6 & 45 & 7,3 & 43 \\
\hline Champán & 89,1 & 525 & 4,9 & 29 & 2,5 & 15 & 3,4 & 20 \\
\hline Vermut & 89,6 & 528 & 4,9 & 29 & 2,4 & 14 & 2,9 & 17 \\
\hline Litrona (entre 2) & 76,4 & 450 & 11,7 & 69 & 5,9 & 35 & 5,9 & 35 \\
\hline Litrona (entre 3) & 84,6 & 498 & 6,3 & 37 & 5,3 & 31 & 3,9 & 23 \\
\hline Litrona (entre 4) & 84,2 & 496 & 2,9 & 17 & 7,6 & 45 & 5,3 & 31 \\
\hline Litrona (entre >4) & 70,3 & 414 & 3,7 & 22 & 6,8 & 40 & 19,2 & 113 \\
\hline Cubalitro (entre 2) & 76,9 & 453 & 13,1 & 77 & 3,6 & 21 & 6,5 & 38 \\
\hline Cubalitro (entre 3) & 86,6 & 510 & 4,6 & 27 & 5,1 & 30 & 3,7 & 22 \\
\hline Cubalitro (entre 4) & 84,2 & 496 & 3,6 & 21 & 6,5 & 38 & 5,8 & 34 \\
\hline Cubalitro (entre >4) & 88,6 & 521 & 1,7 & 10 & 1,5 & 9 & 8,2 & 48 \\
\hline Sidra & 85,3 & 501 & 1,2 & 7 & 2,0 & 12 & 11,4 & 67 \\
\hline
\end{tabular}

Tabla 6. Prueba de comparación de medias de la Cantidad de Alcohol semanal consumida en la muestra de intervención en la primera encuestación (Pre) y a los dos, siete y doce meses de la intervención.

\begin{tabular}{|c|c|c|c|c|c|}
\hline CONSUMO GLOBAL DE ALCOHOL & g.l. & Media & D.T. & $\mathrm{t}$ & p (bilateral) \\
\hline Pre-Post 2 meses & 109 & 2727 &, 4510 & $6,342^{*}$ &, 000 \\
\hline Pre-Post 7 meses & 99 & ,2918 &, 4880 & $5,969^{*}$ &, 000 \\
\hline Pre-Post 12 meses & 105 &, 3124 &, 4644 & $6,926^{*}$ &, 000 \\
\hline Post 2 meses-Post 7 meses & 101 &, 0115 & ,2196 &, 530 &, 597 \\
\hline Post 2 meses-Post 12 meses & 109 & ,0492 & ,2468 & $2,091^{* * *}$ &, 039 \\
\hline Post 7 meses-Post 12 meses & 108 & 0410 & ,2130 & $2,127^{* * *}$ & ,036 \\
\hline
\end{tabular}

Primera encuestación (Pre), dos meses (Post1), siete meses (Post2), doce meses (Post3). ${ }^{*} p<.001,{ }^{* *} p<.01,{ }^{* * *} p<.05$

Con objeto de contrastar el supuesto sobre la previsión de reducción de la cantidad de alcohol semanal consumida en la implementación del programa educativo-preventivo, en los distintos momentos temporales en que fue evaluado, se ha realizado una prueba de comparación de medias para muestras relacionadas de acuerdo a clusters de consumo, cuyos resultados se exponen en la Tabla 6 . Se confirma la eficacia de la intervención en la reducción de la cantidad global de alcohol semanal ingerido en las correspondientes comparaciones entre los hábitos de consumo de los adolescentes seleccionados en la primera recogida de datos antes del programa de intervención y en las correspondientes evaluaciones (dos, siete y doce meses), así como en comparaciones intermedias (a excepción de la comparación entre los dos y siete meses) se mantienen estas reducciones significativas en la cantidad global de alcohol consumido por los adolescentes que participaron en el programa didáctico-preventivo.

\section{DISCUSIÓN}

El estudio descriptivo de los indicadores de actitud hacia la experimentación con alcohol por parte de los adolescentes que han participado en la experiencia didáctico-preventiva, en conjunto, ofrece los resultados siguientes: a) la evaluación de aspectos valorativos de las actitudes hacia el consumo de alcohol (concienciación de los adolescentes ante tal problemática, procesos de sensibilización, análisis de inclinaciones comportamentales y creencias acerca de sus efectos nocivos, etc.) confirma que, en general, los adolescentes encuestados presentan una buena concienciación acerca de la nocividad del consumo abusivo de alcohol, si bien manifiestan deseos de experimentación motivados por la curiosidad y el modelado grupal;

b) aparecen indicios de permisividad ante la experimentación con alcohol en un tercio de los adolescentes de catorce y quince años, e incluso conformidades ante la posibilidad de habituación alcohólica, aunque en general se muestra una disposición de resistencia frente al consumo;

c) el análisis de los aspectos informativos (creencias, conocimientos, opiniones, percepciones de riesgo, etc.) relacionados con el consumo de alcohol corrobora que los adolescentes poseen una adecuada información sobre los efectos derivados de la experimentación, lo cual no tiene por qué redundar en hábitos saludables; finalmente,

d) el análisis de los hábitos de consumo de alcohol confirma el hallazgo de una tendencia extendida hacia la experimentación de fin de semana, obtenida al evaluar la frecuencia de alcohol ingerido regularmente cada semana, concentrándose en una periodicidad de uno a tres dias por semana, 
aunque un porcentaje, que fluctúa entre el quince y el veinte por ciento de los adolescentes, reconocen consumir a diario. Tales resultados van en la línea de los obtenidos por el Eurobarómetro (2007), de acuerdo a los perfiles etiopatogénicos descritos.

La implementación del programa educativo-preventivo a los cuatro grupos experimentales (Intervención Familiar, Grupo Experto, Grupo Informativo y Grupo de Sensibilización básica), y a partir de la evaluación comparativa de sus efectos en diversos períodos temporales, ofrece como consideraciones relativas a mejoras actitudinales y reducciones de consumo de alcohol las siguientes:

a) la confirmación de la eficacia preventiva de la intervención al promoverse cambios favorables en las actitudes hacia el consumo de alcohol en la dirección de desarrollar y/o potenciar actitudes de resistencia ante la experimentación, lo cual se confirma en las cuatro modalidades del programa y su tendencia en las sucesivas evaluaciones;

b) el fortalecimiento de la tendencia de resistencia frente a la experimentación con alcohol, manifestado a través de la evaluación del factor Disposición global hacia el consumo de alcohol, en el Grupo de Sensibilización básica a los dos meses de finalizar el programa de intervención y en el Grupo de Intervención familiar en la segunda evaluación de los resultados, si bien se apunta la mayor dificultad en modificar el componente comportamental de las actitudes que el que explora las creencias y percepciones valorativas;

c) la confirmación de las mejoras preventivas manifestadas en el Grupo de Intervención Familiar en el factor Creencias e Información global sobre el alcohol y sus efectos con un fortalecimiento del componente cognitivo de la actitud manifestado a través de mayores percepciones de riesgo; y, por último,

d) la tendencia a la modificación de hábitos malsanos de experimentación con alcohol, confirmados mediante la reducción significativa de la frecuencia y cantidad de consumo de alcohol por semana en las sucesivas evaluaciones de los resultados en los adolescentes que participaron en el programa didáctico-preventivo.

En consecuencia, se ha comprobado la extensión a un colectivo cada vez más joven de una modalidad de experimentación con alcohol como esparcimiento compartido, de modo que a pesar de promulgaciones legislativas y sancionadoras de semejantes usos en (pre)adolescentes, se ha constatado una estabilización de la edad de inicio al consumo, corroborándose la tendencia hallada en otros estudios con adolescentes españoles (DGPNSD, 2003, 2005, 2007).

Los principales hallazgos obtenidos en los grupos experimentales en la fase previa a la programación educativopreventiva han confirmado la tendencia a una percepción de riesgo distorsionada, acorde con los resultados obtenidos por Moral y Ovejero (2005a) o Pascual (2002), entre otros. De este modo, las opiniones de los adolescentes en riesgo percibido son indicativas de una mayor permisividad ante ciertos usos/abusos de alcohol, de una inclinación más favorable a la experimentación y de una disposición para el consumo más permisiva, dada la implicación de una variable moduladora, como es el nivel de edad, de modo que se han de interpretar apelando a la constatación de una mayor tendencia a la experimentación como indicador de un mayor riesgo percibido (Moral y Ovejero, 2005b; Torregrosa, Inglés, Delgado, Martinez-Monteagudo y Garcia-Fernández, 2007). En este estudio, a partir del análisis del componente comportamental de las actitudes hacia el consumo de alcohol, esto es las inclinaciones para actuar de un modo $u$ otro asociadas a una valoración favorable o desfavorable hacia el objeto de actitud (consumo de alcohol), ha de extraerse la idea relativa a que se refuerza una distorsión de la percepción de riesgo asociada a la falacia a la que ya se ha hecho referencia (droga legal-blanda/ilegaldura, usos lícitos e indebidos) y a su raigambre sociocultural (Ovejero, 2007; Sánchez-Carbonell, 2004) que constituye el pilar sobre el que se fundamenta y potencia la doble moral vinculada al problema de la droga. En todo caso, la relación entre actitud y conducta es sumamente compleja, de modo que tanto la correlación entre ambas como la determinación de una sobre otra es una cuestión controvertida.

Por otro lado, si bien se ha confirmado una tendencia favorable a la concienciación ante los riesgos inherentes al consumo de alcohol, a partir del análisis de los aspectos informativos (creencias, conocimientos, opiniones, percepciones de riesgo, etc.) se ha comprobado que, aunque los adolescentes poseen una información suficiente sobre los efectos derivados de la experimentación, se incide en una percepción de riesgo distorsionada acorde con la mentalidad del usuario (Echeburúa y Del Corral, 1988).

El análisis de las actitudes bajo una triple dimensionalidad (cognitiva, afectiva y reactivo) confirma tendencias de respuesta acordes con la sensibilización de los adolescentes ante la problemática que representa el consumo de alcohol y sus consecuencias, si bien se constata que tales percepciones valorativas denotativas de concienciación básica no se corresponden con disposiciones conductuales de rechazo de la experimentación que vayan acorde con las creencias explicitadas. Baste recordar que actitud y conducta se vinculan de un modo singular, ya que una disposición conductual de rechazo del consumo no necesariamente se refleja en una conducta concreta de rechazo manifiesto, y ésta puede fundamentarse en otros muchos determinantes aparte de en la esfera actitudinal, tendencia que en drogas se ha venido confirmando (Casas, 1998; Carrasco, 2000; Moral y Ovejero, 2005a, 2005b).

Asimismo, se ha confirmado la eficacia preventiva del programa de intervención psicosocial de carácter socioeducativo en las cuatro modalidades, concentrándose a corto y medio plazo (dos y siete meses de finalizar la programación de actividades) el mayor impacto en el cambio actitudinal de los grupos experimentales (Grupo de Intervención Familiar, Experto, Informativo y de Sensibilización básica) en el sentido de un fortalecimiento de las actitudes contrarias al consumo tras la intervención. Este hallazgo se ve confirmado por la tendencia a que las actitudes (creencias, valoraciones, percepciones de riesgo, etc.) y disposiciones hacia el consumo de alcohol de mayor permisividad se vayan afianzando en el Grupo Control en las sucesivas evaluaciones de los resultados, indicativo de la tendencia "natural" en los adolescentes con los que no se 
ha intervenido. Asimismo, en las evaluaciones realizadas con un intervalo de un año de la intervención educativa-preventiva el fortalecimiento actitudinal se mantiene en el Grupo de Intervención Familiary el de Sensibilización básica. En su conjunto, ha de apuntarse la mayor eficacia de los programas capacitadores y de entrenamiento en competencias básicas, en relación aquéllos meramente informativos, lo cual abunda en los resultados hallados en otras investigaciones en las que se incide en la necesidad de involucrar activamente tanto a la familia (Fernández y Secades, 2002; Kumpfer y Johnson, 2007) como al propio grupo de iguales empleados como mediadores (Moradillo, 2003) a modo de educación de calle (González Collantes, 2002), orientando la intervención hacia programas multimodales.

Los resultados, de esta manera, nos llevan a concluir y a observar la necesidad de:

a) comprometernos en labores preventivas de educación primaria y secundaria dentro del aula, que representan una posibilidad de intervención nada desdeñable en adolescentes escolarizados, con programaciones socioeducativas de indole informativo y capacitador que se sirvan de escenarios y vinculaciones psicosociales priorizados y que, idealmente, impliquen en este reto a la propia comunidad.

b) potenciar el entrenamiento en habilidades para la vida, de acuerdo a la recomendación de Botvin (1995) o de Becoña (2007), así como de estrategias de carácter globalizador que abunden en el fortalecimiento de mediadodores preventivos y en el desarrollo de intervenciones multimodales (Hansen, 1995; Werch, 1995).

c) implementar propuestas con mayor implicación y proyección sociocomunitaria en materia de prevención escolar en las que se contemple, ya sea tanto la labor de mediación en el grupo de iguales (Calleja, 2003) como de optimización del tiempo libre y propuestas de ocio alternativo (Ordóñez, 2003), dentro de una programación enriquecedora desarrollada desde la pedagogía del ocio, sobre la que se fundamentada el análisis de Cuenca $(2004,2006,2007)$.

En consecuencia, las propuestas como las implementadas en nuestra investigación de carácter educativo-preventivas con proyección comunitaria parecen permitir enriquecer la formación de habilidades de afrontamiento de los jóvenes, al mismo tiempo que tratan de dotarles y/o potenciar de oportunidades de elección/rechazo y de pautas de actuación saludables con respuestas alternativas, transmitiendo unos contenidos de educación para la salud integrados dentro de su rutina académica y posibilitando (re)integrarles a una comunidad de ciudadanos más cohesionada y participativa. Para ello, tales medidas han de estar insertadas en unas políticas preventivas conjuntas de carácter prospectivo que planteen como estrategias de actuación responder de un modo comprehensivo a la problemática del uso/abuso del alcohol por parte de los jóvenes como reto sociosanitario.

\section{REFERENCIAS}

Anderson, P (2007). Binge drinking in Europe. London: Institute of Alcohol Studies. http://www.dhs.de/web/dhs_international/php/ Reviews/PHPReport_on_binge_drinking.pdf Consultado el 3 de marzo de 2009.

Arnau, M. (2001). Cambios en la edad de inicio del tratamiento de la dependencia alcohólica. ¿Debemos cambiar la oferta asistencial? Adicciones, 13, 139-146.

Bach i Bach, L. (2000). La falta de conciencia del daño alcohólico, firme impedimento para la prevención primaria y secundaria del alcoholismo. Revista Española de Drogodependencias, 25, 114117.

Becoña, E. (2005). Adicción a nuevas sustancias psicoactivas. Psicología Conductual, 13, 349-370.

Becoña, E. (2007). Bases psicológicas de la prevención del consumo de drogas. Papeles del Psicólogo, 28, 11-20.

Becoña, E. y Míguez, M. C. (2006). Consumo de tabaco y alcohol en la población escolar de Galicia. Revista Española de Drogodependencias, 31, 46-56.

Bellis, M. A. y Hughes, K. (2003). Consumo recreativo de drogas y reducción de daños en la vida nocturna global. Adicciones, 15, 289-309.

Botvin, G. J. (1995). Entrenamiento en habilidades para la vida y prevención del consumo de drogas en adolescentes: consideraciones teóricas y hallazgos empíricos. Psicología Conductual, 3, 333-356.

Botvin, G. J. (2000). Preventing Drug Abuse in Schools: Social and Competence Enhancement Approaches Targeting IndividualLevel Etiologic Factor. Addictive Behaviors, 25, 887-897.

Botvin, G. J. y Botvin, E. M. (1992). Adolescent Tobacco, Alcohol and Drug Abuse: Prevention Strategies, Empirical Findings, and Assessment Issues. Developmental and Behavioral Pediatrics, 13, 290-301.

Briñez-Horta, J.A. (2001). Diferencias de género en problemas con el alcohol, según el nivel de consumo. Adicciones, 13, 439-455.

Calafat, A. (2007). El abuso de alcohol de los jóvenes en España. Adicciones, 19, 217-223.

Calafat, A., Fernández, C., Juan, M. y Becoña, E. (2007). Vida recreativa nocturna de los jóvenes españoles como factor de riesgo frente a otros más tradicionales. Adicciones, 19, 125-131.

Calafat, A., Juan, M., Becoña, E., Castillo, A., Fernández, C., Franco, M., Pereiro, C. y Ros, M. (2004). El consumo de alcohol en la lógica del botellón. Adicciones, 17, 193-202.

Calleja, M.F. (2003). Mediadores sociales en el grupo de iguales. Adicciones, 15, 27

Casa, M.J., O'Ferrall, C. y Vaca, F.J. (2001). Evolución del perfil del enfermo alcohólico durante los últimos veinte años. Revista Española de Drogodependencias, 26, 127-134.

Casas, J. (1998). Actitudes, habilidades sociales y autocontrol en la prevención del consumo abusivo de alcohol y tabaco. Tesis Doctoral. Madrid: Universidad Autónoma.

Castillo, G. (1999). El adolescente y sus retos. La aventura de hacerse mayor. Madrid: Pirámide.

Cortés, M. T., Espejo, B. y Giménez, J. A. (2007). Caracteristicas que definen el fenómeno del botellón en universitarios y adolescentes. Adicciones, 19, 357-372. 
Cortés, M. T., Espejo, B. y Giménez, J. A. (2008). Aspectos cognitivos relacionados con la práctica del botellón. Psicothema, 20, 396402.

Cuenca, M. (2004). Pedagogía del Ocio: Modelos y Propuestas. Bilbao: Universidad de Deusto.

Cuenca, M. (Coord.) (2006). Pedagogía del ocio, una aproximación global. Bilbao: publicaciones Universidad de Deusto.

Cuenca, M. (2007). La otra cara del "botellón". Padres y maestros, 305, 43-47.

Delegación del Gobierno para el Plan Nacional sobre Drogas (2003). Indicadores Tratamiento, Urgencias y Mortalidad. Informe año 2001. Madrid: DGPNSD.

Delegación del Gobierno para el Plan Nacional sobre Drogas (2004). Encuesta sobre Drogas a la Población Escolar 2003. Madrid: DGPNSD.

Delegación del Gobierno para el Plan Nacional sobre Drogas (2005a). Encuesta sobre Drogas a la Población Escolar 2004. Madrid: DGPNSD.

Delegación del Gobierno para el Plan Nacional sobre Drogas (2005b). Plan de Acción 2005-2008 de la Estrategia Nacional sobre Drogas. Madrid: DGPNSD.

Delegación del Gobierno para el Plan Nacional sobre Drogas (2006a). Encuesta sobre Drogas a la Población Escolar 2005. Madrid: DGPNSD.

Delegación del Gobierno para el Plan Nacional sobre Drogas (2006b). Secreto de la buena vida. Madrid: DGPNSD.

Delegación del Gobierno para el Plan Nacional sobre Drogas (2006c). Drogas. Hay trenes que es mejor NO coger. Campaña institucional referente a la prevención del uso de drogas. Madrid: DGPNSD.

Delegación del Gobierno para el Plan Nacional sobre Drogas (2007). Informe sobre el alcohol en España. Madrid: DGPNSD.

Echeburúa, E. y De Corral, P. (1988). Evaluación y tratamiento de un caso clínico de alcoholismo. En D. Macià y F. X. Méndez. Aplicaciones clínicas de la evaluación y modificación de conducta. Estudio de Casos (pp. 147-207). Madrid: Pirámide.

Espada, J. P., Méndez, F. X. e Hidalgo, M. D. (2000). Consumo de alcohol en escolares: descenso de la edad de inicio y cambios en los patrones de ingesta. Adicciones, 12, 57-64.

Espada, J. P., Méndez, X., Griffin, K. y Botvin, G. J. (2003). Adolescencia: consumo de alcohol y otras drogas. Papeles del Psicólogo, 84, 9-17.

Eurobarómetro (2007). Attitudes towards Alcohol. http://www.ec.europa. eu/health/ph_determinants/life_style/alcohol/documents/ebs272_ en.pdf. Consultado el 3 de marzo de 2009.

Farke, W. y Anderson, P. (2007). El consumo concentrado de alcohol en Europa. Adicciones, 19, 333-339.

Fernández, J. R. y Secades, R. (Dirs.) (2002). Intervención familiar en la prevención de las drogodependencias. Madrid: Ministerio de Interior. Secretaria General Técnica. Plan Nacional sobre Drogas.

Franco, M., Juan, M., Pereriro, C., Calafat, A., Castillo, A. y Becoña, E. (2005). El consumo de alcohol en la lógica del botellón. Adicciones, 17, 193-203.

Gómez-Fraguela, J., Fernández, N., Romero, E. y Luengo, A. (2008). El botellón y el consumo de alcohol y otras drogas en la juventud. Psicothema, 20, 211-217.

González Collantes, L.A. (2002). Educación de calle. Idea Prevención, $24,18$.

Haddock G. y Zanna, M. (1999). Cognition, affect and the prediction of social attitude. En S. Stroke y M. Hewston (Eds.). European
Review of Social Psychology, Vol 10. Cambridge: Cambridge University Press.

Hansen, W. B. (1995). Aproximaciones psicosociales a la prevención el uso de las investigaciones epidemiológicas etiológicas para el desarrollo de intervenciones efectivas. Psicología Conductual, 3, 357-378.

Kumpfer, K. L. y Johnson, J. L. (2007). Intervenciones de fortalecimiento familiar para la prevención del consumo de sustancias en hijos de padres adictos. Adicciones, 19, 13-25.

Legaza, I. (2002). Programa Comunitario de Prevención de Drogodependencias. Idea Prevención, 24, 24.

Macià, D. (1986). Cuestionario de Actitudes acerca del alcohol y Cuestionario de Información. Método Conductual de Prevención de Drogodependencias. Valencia: Promolibro.

Macià, D. (1995). Método conductual de prevención de la drogodependencia. Valencia: Promolibro.

Macià, D. y Méndez, F. X. (1988). Aplicaciones clínicas de la evaluación y modificación de conducta. Estudio de Casos. Madrid: Pirámide.

Martín, E. y Moncada, S. (2003). Programa de prevención de ocio alternativo. Adicciones, 15, 327-346.

Miguez, M. C. y Becoña, E. (2008). ¿Consomen alcohol os nosos rapaces? Revista galega do ensino, 52, 79-84.

Monrás, M. (2001). Cambios en la edad de inicio del tratamiento de la dependencia alcohólica. ¿Debemos cambiar la oferta asistencial? Adicciones, 13, 139-146.

Moradillo, F. (2003). La educación sobre drogas entre alumnos en un centro de Educación Secundaria. Adicciones, 15, 63-64.

Moral, M. V. (2007). Cambios en las representaciones sociales sobre las drogas y sus usuarios en la sociedad española. SMAD Revista Electrónica Salud mental, Alcohol y Drogas, 3, 1-15.

Moral, M. V. y Ovejero, A. (2004). Jóvenes, globalización y postmodernidad: Crisis de la adolescencia social en una sociedad adolescente en crisis. Papeles del Psicólogo, 25, 72-79.

Moral, M.V. y Ovejero, A. (2005a). Modificación de las actitudes, los hábitos y frecuencia de consumo de alcohol y otras sustancias psicoactivas en adolescentes españoles a partir de un programa educativo-preventivo. Revista Colombiana de Psicología, 14, 100-118.

Moral, M. V. y Ovejero, A. (2005b). Análisis diferencial por niveles de edad de las actitudes hacia el consumo de sustancias psicoactivas en adolescentes españoles. Interamerican Journal of Psychology, 39, 325-338.

Moral, M. V., Rodríguez, F. J. y Sirvent, C. (2005). Motivadores de consumo de alcohol en adolescentes: Análisis de las diferencias intergénero y propuesta de un continuum etiológico. Adicciones, 17, 105-120.

Moral, M. V., Rodriguez, F. J. y Sirvent, C. (2006). Factores relacionados con las actitudes juveniles hacia el consumo de alcohol y otras sustancias psicoactivas. Psicothema, 18, 52-58.

Newcomb, T. M. (1964). Manual de Psicologia Social. Buenos Aires: Eudeba.

Ordóñez, A. (2003). Programas desarrollados en el ámbito del tiempo libre. Adicciones, 15, 70.

Ovejero, A. (2000). La adicción como búsqueda de identidad: una base teórica psicosocial para una intervención eficaz. Intervención Psicosocial, 9, 35-48.

Ovejero, A. (2007). Adicciones y contexto sociocultural: perspectivas psicosociológicas críticas. Revista Española de Drogodependencias, 3, 292-309. 
Pascual, F. (2002). Percepción del alcohol entre los jóvenes. Adicciones, $14,123-132$

Pons, J. y Buelga, S. (1995). Estructura de valores y abuso de alcohol en adolescentes. En J. A. Conde y A. I. Isidro (Comps.). Psicología Comunitaria, Salud y Calidad de Vida (pp. 305-313). Madrid: Eudema.

Sánchez-Carbonell, X. (2004). Contexto cultural y consecuencias legales del consumo de drogas. Trastornos Adictivos, 6, 1-4.

Santacreu, J., Froján, M. X. y Hernández, J. A. (1990). AlCA: Autoinforme sobre Consumo de sustancias psicoactivas. Departamento de Psicología de la Salud. Universidad Autónoma de Madrid.

Santacreu, J. y Froján, M. X. (1994). Evaluación del consumo de drogas. En R. Fernández-Ballesteros. Evaluación conductual hoy. Un enfoque para el cambio en psicología clínica y de la salud (pp. 571-612). Madrid: Pirámide.
Sirvent, C., Moral, M. V. y Rodríguez, F. J. (2007). Jóvenes \&t Alcohol: Conjunto didáctico-audiovisual para educadores, psicólogos, padres y colectivos mediadores. Valencia: Nau Llibres.

Torregrosa, M. S., Inglés, C. J., Delgado, B, Martinez-Monteagudo, M. C. y Garcia-Fernández, J. (2007). Frecuencia del consumo de drogas legales: diferencias de edad en la adolescencia. Revista Española de Drogodependencias, 2, 181-195.

Vega, A. (2003). Repensar la educación sobre las drogas para una nueva prevención. Revista Española de Drogodependencias, 28, 7-23.

Werch, Ch. E. (1995). Prevención del consumo de alcohol, tabaco y otras drogas entre los jóvenes: recomendaciones para los futuros programas preventivos. Psicología Conductual, 3, 379-394. 
